\title{
Taxonomic Studies on the Genus Athesapeuta (Coleoptera: Curculionidae: Baridinae) from India with Description of Three New Species
}

\author{
B. Ramesha and V. V. Ramamurthy \\ Division of Entomology, Indian Agricultural Research Institute, New Delhi 110012, India \\ Correspondence should be addressed to B. Ramesha, b.ramesha@gmail.com \\ Received 20 September 2011; Revised 19 November 2011; Accepted 19 November 2011 \\ Academic Editor: Arthur G. Appel
}

Copyright ( $) 2012$ B. Ramesha and V. V. Ramamurthy. This is an open access article distributed under the Creative Commons Attribution License, which permits unrestricted use, distribution, and reproduction in any medium, provided the original work is properly cited.

\begin{abstract}
Six species of genus Athesapeuta Faust (Coleoptera: Curculionidae: Baridinae) from India and the adjacent countries are included, of which three new species, namley meghalayensis sp. nov., richardi sp. nov., and spinulatus sp. nov., are described. An annotated checklist of known species along with their synonymy and distribution is given. Descriptions are supplemented with details of genitalia and elytral vestiture; a key to the species studied is provided.
\end{abstract}

\section{Introduction}

Athesapeuta belongs to the tribe Madarini which at present is considered under the subfamily Baridinae of the family Curculionidae [1]. The majority of its species are known from the Oriental region, of which eight are from India. Except for the studies by Faust [2, 3] and Marshall [4], taxonomic studies on the Indian fauna are inadequate, scattered, and need to be updated and supplemented with descriptions of genitalia, morphometrics, and terminology. In particular, elytral vestiture and female genitalia which are emerging as important characters have not been included in descriptive work. The present study addresses these gaps for the currently recognized species and adds three new species.

\section{Materials and Methods}

Voucher specimens and type material are deposited with the National Pusa Collection (NPC) of the Division of Entomology, Indian Agricultural Research Institute, New Delhi. Materials from the National Pusa Collection (NPC) of the Division of Entomology, Indian Agricultural Research Institute, New Delhi; Forest Research Institute (FRI), Dehra Dun; Zoological survey of India (ZSI), Kolkotta; Panjab University (PU), Chandigarh, were included. Paratypes were studied for all the species described, and they are compared with original literature and photographs. All taxonomic characters, except the elytral vestiture and genitalia, were studied in intact specimens. Specimens were processed following Supare et al. [5], and genitalia and elytral vestiture were studied following Ramamurthy and Ghai [6]. The terminology of Supare et al. [5], Thompson [7], Poorani and Ramamurthy [8], O'Brien and Pakaluk [9], Wanat [10], and Davis [11] was followed for the description of female and male genitalia. General taxonomic characters and genitalia were studied with Leica M205FA stereozoom microscope, and elytral vestiture was studied with Leica DM1000 phase contrast microscope. Photographs were captured using the software Leica application Suite ver. 2.8.2 on a Leica DFC290 camera. Illustrations were made using a drawing tube fitted with a camera lucida and scales of magnification provided in the illustrations. Total length given in the descriptions is excluding rostrum, and the standard length is from anterior margin of pronotum to end of pygidium. The measurements given in the descriptions are mean and standard error except for new species, wherein measurements are of holotype.

\section{Genus: Athesapeuta Faust}

Athesapeuta Faust 1894 [2]. Type species: Baridius subsignatus Motschulsky, 1866 [12] = Athesapeuta motschulskyi Voss, 1958 [13]; gender: Female. (By “Original Designation”). 
Description. General colour shiny black; body rhomboidal, subcylindrical, or oblongovate, much longer than broad. Head not separated from rostrum by a deep transverse incision, frons narrower than base of rostrum, 1.5-4x as broad as long. Rostrum long, cylindrical, straight at least in basal half, separated from head by a sharp sulcus between eyes; more or less curved with mandibles adducent type, dentate internally or conical with straight cutting edge, 0.79 $0.98 \mathrm{x}$ as long as head and pronotum combined (Figure 1(a)); eyes $1.28-2.12 \mathrm{x}$ as wide as long. Antennae inserted at 1.1$1.8 \mathrm{x}$ of length from base of rostrum; scape $6.25-10 \mathrm{x}$ as long as broad; funicle widening distally; seventh funicle not fitting closely to club (Figure 1(b)). Prothorax generally shallowly bisinuate at posterior dorsal margin, anterior dorsal margin truncate, not produced over head when viewed in profile, as broad as to $1.28 \mathrm{x}$ as broad as long. Scutellum generally trapezoidal, as broad as to $1.5 \mathrm{x}$ as broad as long. Elytra oblong or ovate, smooth; $0.5-0.92 \mathrm{x}$ as wide as prothorax; separately arcuate at base; deeply striate, striae 10 complete; separately rounded at apices, with narrow membrane and paler fringe along apical margin, without any trace of subapical calli (Figure 1(c)). Legs with femora clavate (Figure 1(d)), unarmed, not sulcate beneath; tibiae with longitudinally confluent punctures, sulcate, not tuberculate (Figure 1(e)); claws almost parallel to each other, more or less divergent, narrowly separated (Figure 1(f)). Sternum with a furrow on prosternum; procoxa $1.53-2.5 \mathrm{x}$ as wide as intercoxal process; hind margin of prosternum produced in middle; mesosternum transversely impressed, intercoxal process $0.9-1.25 \mathrm{x}$ as broad as a coxa; metasternum elongate, 1.5-1.66x as long as mesocoxa; pro-, meso-, and metasterna not interrupted in continuity by mesosternum (Figure $1(\mathrm{~g})$ ). Abdomen with first and second ventrites not connate; pygidium fully or broadly exposed, directed posterioventrally, visible dorsally, without transverse carina along hind margin of elytra in general; lateral part of fifth ventrite visible dorsally on each side of pygidium. Female genitalia with spermatheca more or less sclerotised at distal arm, distal arm shorter than proximal arm; spiculum ventrale $1.5-2.5 \mathrm{x}$ as long as basal plate; apical end with hairs. Male genitalia with median lobe moderately sclerotised; apophyses $0.25-1.06 \mathrm{x}$ as long as median lobe.

\section{Annotated Checklist}

Faust [2] described this genus, and Voss [13] synonymised Baridius subsignatus with Athesapeuta motschulskyi, the type species. The majority of species were described before 1950 . Faust contributed the most with nine species, followed by Marshall (7), Voss (6), Bohemann and Hustache (3 each); Motschulsky (2), and Fabricius, Gerstaecker and Zimmerman (1 each). This genus is predominantly Oriental (18 species) Thirteen are Afrotropical, and five occur in the Palaearctic.

Checklist: Type species: Baridius subsignatus Motschulskyi, 1866 [12] = Athesapeuta motschulskyi Voss, 1958 [13]. (For more details see Table 1).

\section{Description of Species}

5.1. Athesapeuta cyperi Marshall, 1928 [17]. (Figures 2(a), 3(a), 4(a), 5(a), 6(a), 6(b), 8(a), 9(a), 10(a), 11(a), 11(d), 12(a), 13(a)-13(c), 14(a), 14(b), 15(a)-15(d) and 16(a)$16(\mathrm{c}))$.

Description. Colour shiny black; antennae, tibiae, elytra on lateral and apical margins reddish brown. Head bare, finely alutaceous with sparse punctures, $1.5 \mathrm{x}$ as broad as long; eyes $1.28 \mathrm{x}$ as wide as long. Rostrum $0.79 \mathrm{x}$ as long as head and pronotum combined, strongly curved, smooth, with four rows of small punctures in addition to punctate area above scrobes, sparse vestiture on each side of base, at middle $0.77 \mathrm{x}$ as broad as at apex, $0.76 \mathrm{x}$ as broad as at base (Figures 2(a) and 3(a)). Antennae inserted at 1.57x of length from base of rostrum; scape slender, long, 9.13x as long as broad, impunctate; funicle with first segment $3.5 \mathrm{x}$ as long as second and third combined, third $0.5 \mathrm{x}$ as long as broad, segments three to seven slightly transverse, subequal in length and breadth; funicle $1.18 \mathrm{x}$ as long as club (Figure 4(a)). Prothorax 1.03x as broad as long, sides subparallel from base to middle, anterior margin truncate, behind apex with tubular constriction, posterior margin shallowly bisinuate, at middle $1.91 \mathrm{x}$ as broad as at apex, and $0.98 \mathrm{x}$ as broad as at base; dorsum gently convex longitudinally, set with close shallow separated punctures, with a broad impunctate median stripe, punctures on pleurae larger and subreticulate (Figure 5(a)). Scutellum bare, trapezoidal, with two low longitudinal costae, $1.09 \mathrm{x}$ as broad as long. Elytra oblong ovate, separately rounded at apex, at middle $1.14 \mathrm{x}$ as broad as at apex, $1.13 \mathrm{x}$ as broad as at base; striae deep, indefinitely punctate, not diminishing at apex, striae 10 complete; intervals flat, $3 \mathrm{x}$ as broad as a striae, with a row of large shallow punctures, each with minute recumbent scale, vestiture small on intervals one to five, larger, scalelike on outer intervals (Figure 8(a)); elytral vestiture whitish, rod-shaped, tapering and pointed at base, blunt at apex, surface with striations reaching apex (Figure 9(a)). Legs coarsely punctate, each puncture containing a grey elongate vestiture; tibia sulcate; profemur with a fringe of long vestiture on ventral surface (Figure 6(a)), 1.12x as long as mesofemur, 1.09x as long as metafemur. Protibia $1.53 \mathrm{x}$ and $1.25 \mathrm{x}$ as long as meso- and metatibia, respectively, with a sharp tooth on inner edge at about middle in males (Figure 6(b)), females without it. First tarsal segment 1.1x as long as broad, $1.1 \mathrm{x}$ as long as second, $0.83 \mathrm{x}$ as long as and $0.71 \mathrm{x}$ as broad as third, third $1.16 \mathrm{x}$ as broad as long, fourth $3 \mathrm{x}$ as long as broad. Prosternum with deep transverse sulcus behind apex, base with raised fovea. Procoxa 1.87x as broad as its intercoxal process; mesosternum plate-like, depressed at base, raised at apex, intercoxal process $0.96 \mathrm{x}$ as broad as mesocoxa; metasternum depressed in middle with longitudinal impressed line, intercoxal process $1.33 \mathrm{x}$ as broad as metacoxa. Venter black, strongly punctate, each with broad vestiture; anterior margin of first ventrite broadly and shallowly ogival, posterior margin straight, $2.92 \mathrm{x}$ as broad as long, $1.74 \mathrm{x}$ as long as second, second $5 \mathrm{x}$ as broad as long, $0.65 \mathrm{x}$ as long as three and four combined, ventrites third and fourth subequal in length, five $2.18 \mathrm{x}$ as broad as long, 
TABle 1

\begin{tabular}{|c|c|c|}
\hline Sl No & Species & Distribution \\
\hline (1) & affinis Faust, 1898 [3] & India \\
\hline$(2)$ & amoena Voss, 1958 [13] & China \\
\hline (3) & armata Hustache, 1932 [14] & Madagascar \\
\hline (4) & atronuda Marshall, 1941 [15] & Uganda \\
\hline$(5)$ & aurantiaca Faust, 1894 [2] & Myanmar \\
\hline$(6)$ & bengalica Faust, 1894 [2] & India \\
\hline$(7)$ & chinensis Faust, 1894 [2] & China \\
\hline (8) & conradti Hustache, 1932 [16] & Cameroun \\
\hline (9) & cyperi Marshall, 1928 [17] & Philippines \\
\hline$(10)$ & dodonis (Marshall) = Baris dodonis Marshall, 1936 [18]; Pajni and Kohli, 1990 [19] & Uganda \\
\hline$(11)$ & $\begin{array}{l}\text { famula (Fabricius) = Curculio famula Fabricius, } 1798[20] \text {; Hustache, } 1938[21]=\text { Rhynchaenus } \\
\text { famula (Fabricius, } 1798[20]) ; \text { Fabricius, } 1801[22] ; \text { Hustache, } 1938[21]=\text { Baridius famula } \\
\text { (Fabricius, } 1798[20]) ; \text { Boheman in Schoenherr, } 1836[23] ; \text { Hustache, } 1938[21]= \\
\text { centrodentatus Desbrochers des Loges, } 1891[24] ; \text { Hustache, } 1938[21]\end{array}$ & India \\
\hline$(12)$ & $\begin{array}{l}\text { flavicornis (Boheman in Schoenherr) = Baridius flavicornis Boheman in Schoenherr, } 1836 \text { [23]; } \\
\text { Hustache, } 1938[21]\end{array}$ & USA \\
\hline$(13)$ & gyrosicollis Marshall, 1948 [25] & Southern Shan States \\
\hline$(14)$ & immaculata Faust, 1898 [3] & India \\
\hline$(15)$ & latifasciata Voss, 1958 [13] & China \\
\hline (16) & $\begin{array}{l}\text { lineolatofasciata (Motschulsky) = Baridius lineolatofasciata Motschulsky, } 1866 \text { [12]; Faust, } 1894 \\
\text { [2] }\end{array}$ & India \\
\hline$(17)$ & madugodana Voss, 1957 [26] & Sri Lanka \\
\hline$(18)$ & meghalayensis sp. nov. & India \\
\hline$(19)$ & motschulskyi Voss, 1958 [13] & China \\
\hline$(20)$ & oryzae Marshall, 1916 [4] & India \\
\hline$(21)$ & pinguis Faust, 1894 [2] & Myanmar \\
\hline$(22)$ & politirostris Voss, 1962 [27] & Congo \\
\hline$(23)$ & richardi sp. nov. & India \\
\hline$(24)$ & sculptilis Gerstaecker, 1871 [27] = scutellaris Faust, 1896: 145 & Africa \\
\hline$(25)$ & secura Faust, 1894 [2] & Myanmar \\
\hline$(26)$ & semirubra (Hustache) = Titanobaris semirubra Hustache, 1935 [28]; Marshall, 1941 [15] & Angola \\
\hline$(27)$ & soror Faust, 1898 [3] & India \\
\hline$(28)$ & spinulatus sp. nov. & India \\
\hline$(29)$ & subcalva Marshall, 1941 [15] & Uganda \\
\hline (30) & $\begin{array}{l}\text { subsignatus (Boheman in Schoenherr) = Baridius subsignatus Boheman in Schoenherr, } 1836 \\
\text { [23]; Faust, } 1894 \text { [2] }\end{array}$ & Africa \\
\hline$(31)$ & $\begin{array}{l}\text { subsignata (Motschulsky) not Boheman = Baridius subsignata Motschulsky, } 1866 \text { [12]; Faust, } \\
1894 \text { [2] }\end{array}$ & India \\
\hline$(32)$ & sculpticollis Voss, $1958[13]$ & China \\
\hline$(33)$ & ulvae Zimmerman, 1942 [29] & Guam \\
\hline$(34)$ & varicolor Marshall, 1941 [15] & Uganda \\
\hline$(35)$ & $\begin{array}{l}\text { versicolor }(\text { Boheman })=\text { Baridius versicolor Boheman, } 1859[30]=\text { Baris versicolor }(\text { Boheman, } \\
1859[30]) ; \text { Hustache, } 1938 \text { [21]; Pajni and Kohli, } 1990[19]\end{array}$ & Indonesia \\
\hline$(36)$ & vinculata Faust, 1894 [2] & Myanmar \\
\hline
\end{tabular}

posterior margin truncate; pygidium distinctly punctate with fringes of vestiture, exposed on ventral side, with an archshaped marking at middle in males (marking being the junction of tergites VII and VIII), females without it, $1.03 \mathrm{x}$ as broad as long (Figure 10(a)).
Female Genitalia. Spermatheca not sclerotised, distal arm as long and as broad as proximal arm, angle between proximal and distal arms not acute, nodulus small, ramus flat, cornu blunt (Figures 11(a) and 14(a)). Spiculum ventrale with shaft elongate, $1.5 \mathrm{x}$ as long as basal plate, basal plate $5 \mathrm{x}$ as long as 


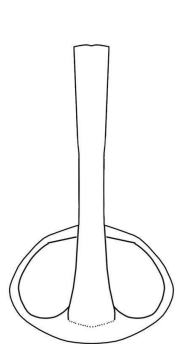

(a)

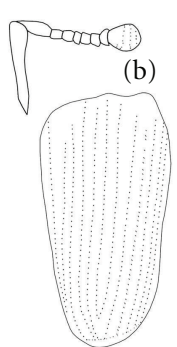

(c)

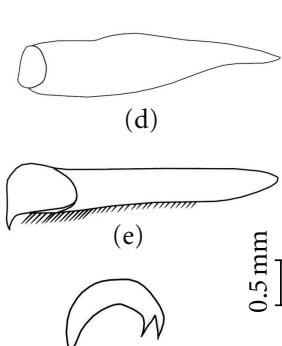

(f)

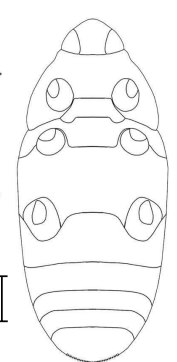

(g)

Figure 1: Athesapeuta. Genus characters: (a), rostrum, dorsal view; (b), antennae; (c), elytron, dorsal view; (d), femur, lateral view; (e), tibiae; (f), tarsal claw; (g), habitus; ventral view.

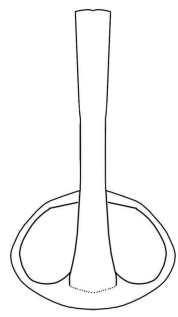

(a)

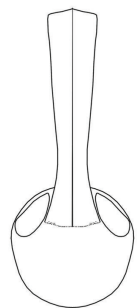

(d)

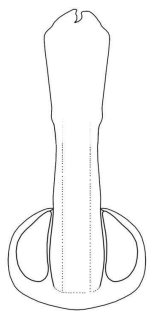

(b)

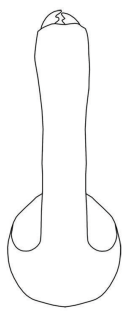

(e)

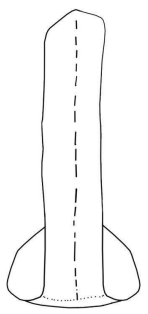

(c)

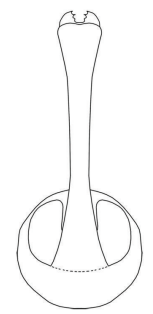

(f)

Figure 2: Rostrum, dorsal view: (a), A. cyperi; (b), A. immaculata; (c), A. meghalayensis sp. nov.; (d), A. oryzae; (e), A. richardi sp. nov.; (f), A. spinulatus sp. nov.

broad, apical end truncate, without hairs (Figures 11(d) and 14(b)).

Male Genitalia. Apophyses $0.25 \mathrm{x}$ as long as median lobe, $0.25 \mathrm{x}$ as long as spiculum gastrale; median lobe moderately sclerotised, parallel sided from base to behind middle, apex sinusoidal, at middle $1.42 \mathrm{x}$ as broad as at apex and as broad as at base (Figures 13(a)-13(c) and 15(a)-15(d)). Spiculum gastrale uniformly thick, curved at apex, $11.6 \mathrm{x}$ as long as broad (Figures 12(a) and 15(d)).

Measurements. Total length: $3.25 \pm 0.23 \mathrm{~mm}$; standard length: $3-3.30 \mathrm{~mm}$; breadth: $1.36 \pm 0.07 \mathrm{~mm}$.

Material Examined. India: $60^{7} 0^{7}$, Maharashtra: Phaltan, feeding on nut sedge, x.1999, Coll. Nimbkar; $40^{7} \sigma^{7}, 3$ 우, West Bengal: Kolkata, 11.i.2011, Coll. Ramasubramanian, larvae boring on Cyperus rotundus.

Distribution. India: Maharashtra; West Bengal. Philippines: Los Banos. Hawaii: Honolulu, Ohau.
5.2. Athesapeuta immaculata Faust, 1898 [3]. (Figures 2(b), 3(b), 4(b), 5(b), 8(b), 9(b), 9(c), and 16(d)-16(f)).

Description. Colour shiny black. Head with sparse punctures, $3.2 \mathrm{x}$ as broad as long; eyes $1.38 \mathrm{x}$ as wide as long. Rostrum $0.98 \mathrm{x}$ as long as head and pronotum combined, strongly curved, with two dorsal carinae from base to antennal insertion and then fading out, at middle $0.85 \mathrm{x}$ as broad as at apex, $0.92 \mathrm{x}$ as broad as at base (Figures 2 (b) and 3(b)). Antennae inserted at $1.8 \mathrm{x}$ of length from base of rostrum; scape brown, impunctate, $8.33 \mathrm{x}$ as long as broad; funicle with first segment $1.16 \mathrm{x}$ as long as second and third combined, third as long as broad, segments three to seven slightly transverse and subequal in length and breadth; funicle $1.81 \mathrm{x}$ as long as club; club ovate (Figure 4(b)). Prothorax as broad as long, with granular punctures, anterior margin truncate, behind the apex without tubular constriction, posterior margin shallowly bisinuate, at middle $1.78 \mathrm{x}$ as broad as at apex, and $0.75 \mathrm{x}$ as broad as at base; dorsum gently convex longitudinally, set with shallow regular punctures, confluent in curves, without any median line. Scutellum raised, trapezoidal, sparsely punctate, as long as 


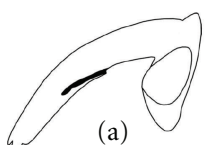

(a)

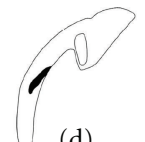

(d)

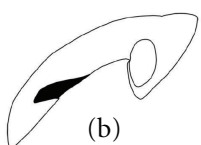

(b)

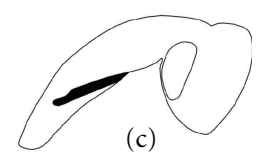

(c)

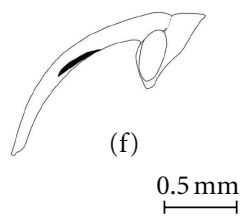

Figure 3: Rostrum, lateral view: (a), A. cyperi; (b), A. immaculata; (c), A. meghalayensis sp. nov.; (d), A. oryzae; (e), A. richardi sp. nov.; (f), A. spinulatus sp. nov.

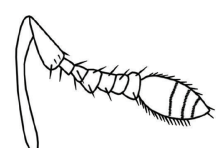

(a)

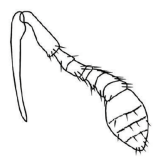

(d)

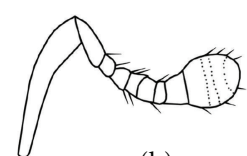

(b)

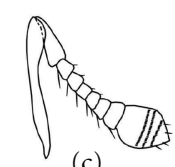

(c)

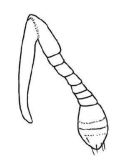

(e)

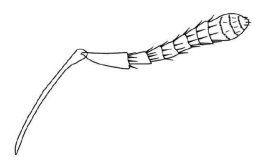

(f)

Figure 4: Antenna: (a), A. cyperi; (b), A. immaculata; (c), A. meghalayensis sp. nov.; (d), A. oryzae; (e), A. richardi sp. nov.; (f), A. spinulatus sp. nov.

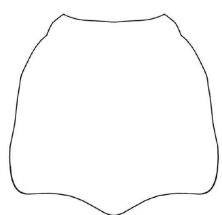

(a)

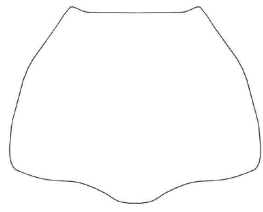

(d)

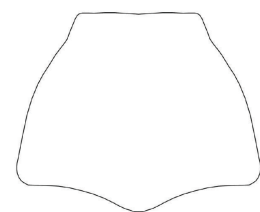

(b)

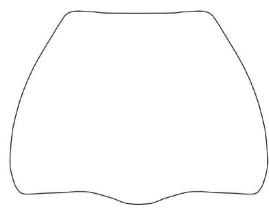

(e)

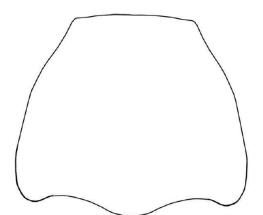

(c)

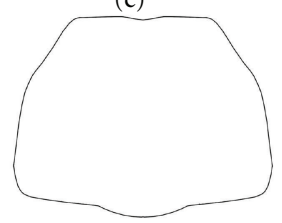

(f)

Figure 5: Prothorax, dorsal view: (a), A. cyperi; (b), A. immaculata; (c), A. meghalayensis sp. nov.; (d), A. oryzae; (e), A. richardi sp. nov.; (f), A. spinulatus sp. nov.

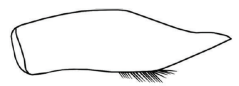

(a)

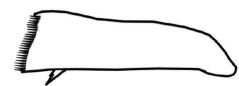

(b)

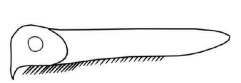

(c)

Figure 6: (a, b) Profemur and metatibia: A. cyperi; (c) Protibia: A. oryzae.

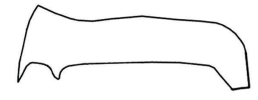

(a)

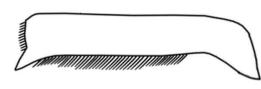

(b)

Figure 7: Protibia and metatibia: A. richardi sp. nov. 


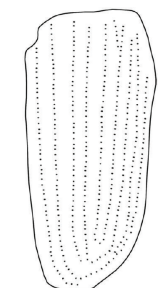

(a)

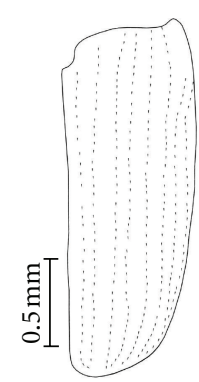

(d)

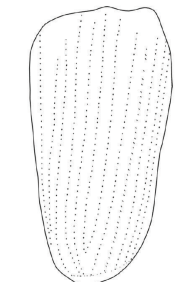

(b)

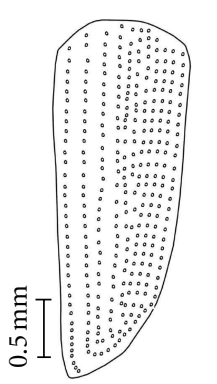

(e)

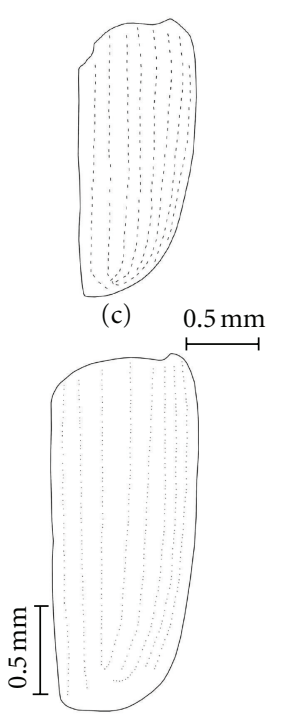

(f)

Figure 8: Elytron, dorsal view: (a), A. cyperi; (b), A. immaculata; (c), A. meghalayensis sp. nov.; (d), A. oryzae; (e), A. richardi sp. nov.; (f), A. spinulatus sp. nov.

broad (Figure 5(b)). Elytra oblong ovate, separately rounded at apex, without any posterior calli, at middle $1.32 \mathrm{x}$ as broad as at apex, $1.22 \mathrm{x}$ as broad as at base; striae shallow, with distant catenulate punctures which do not encroach on intervals, striae 10 complete; intervals flat, $1.5 \mathrm{x}$ as broad as a striae, with a row of transverse punctures, each containing a minute recumbent scale (Figure $8(\mathrm{~b})$ ). Elytral vestiture predominantly of two types; either yellowish white, base tapering and apex concave, surface with granular serrations (Figure 9(b)), or brownish yellow, both ends broader and surface with dense granular serrations (Figure 9(c)). Legs coarsely punctate, each puncture containing grey vestiture; tibia sulcate; profemur $0.90 \mathrm{x}$ as long as mesofemur, $0.83 \mathrm{x}$ as long as metafemur. Protibia $1.2 \mathrm{x}$ and $0.93 \mathrm{x}$ as long as meso- and metatibia, respectively. First tarsal segment $1.66 \mathrm{x}$ as long as broad, $1.66 \mathrm{x}$ as long as second, as long as and $0.54 \mathrm{x}$ as broad as third, third $0.90 \mathrm{x}$ as broad as long, fourth $4 \mathrm{x}$ as long as broad. Prosternum with deep transverse sulcus behind apex, base of prosternum with raised fovea, with definite punctures all over sternum and each punctures with a grey recumbent vestiture. Procoxa $2.5 \mathrm{x}$ as broad as intercoxal process; mesosternum plate like, depressed at base and apex, intercoxal process $1.25 \mathrm{x}$ as broad as mesocoxa; metasternum depressed in middle with longitudinal impressed line, intercoxal process 1.08x as broad as metacoxa.

Measurements. Total length: $5.4-5.5 \mathrm{~mm}$; standard length: $4.7 \mathrm{~mm}$; breadth: $2.2-2.4 \mathrm{~mm}$.

Material Examined. 2 specimens, location unknown, from Nagasilla grass (abdomen damaged), 12.vii.1985, Coll. unknown.

Distribution. India.
5.3. Athesapeuta meghalayensis sp. nov. (Figures 2(c), 3(c), 4(c), 5(c), 8(c), 9(d), 9(e), 10(b), 12(b), 13(d)-13(g), 15(e)15(i), and 16(g)-16(i)).

Diagnosis. It is closely related to A. richardi sp. nov., but differs in prothorax with broad stripe of yellow vestitures (white in $A$. richardi sp. nov.), with a smooth median line (absent in A. richardi sp. nov.); posterior end of tibia does not carry sharp tooth (present in A. richardi sp. nov.), metatibia lateroventrally without fringes of grey hairs (present in $A$. richardi sp. nov.).

Description. Colour black. Head with close regular punctures, $4 \mathrm{x}$ as broad as long; eyes ventrally placed, $2.12 \mathrm{x}$ as wide as long. Rostrum 0.9x as long as head and pronotum combined, strongly curved, gradually widening, irregularly punctate, each punctures with yellow vestiture, more prominent in basal region, irregular punctures become reticulate and rugose beyond antennal insertion, with a median smooth impunctate line, almost parallel sided, without any subbasal dilation (Figures 2(c) and 3(c)), at middle 1.09x as broad as at apex, $0.92 \mathrm{x}$ as broad as at base. Antennae inserted at $1.22 \mathrm{x}$ of length from base of rostrum; scape robust, $6.25 \mathrm{x}$ as long as broad, almost impunctate; funicle with first segment $1.2 \mathrm{x}$ as long as second and third combined, segments second to seven carry sharp spines all over surface, third as long as broad, segments three to seven transverse and subequal in length and breadth; funicle $1.46 \mathrm{x}$ as long as club; club ovate (Figure 4(c)). Prothorax 1.03x as broad as long, with granular punctures, with a stripe of yellow vestiture on lateral aspect just behind the anterior margin on both sides, which is continuous with scaling of lower surface, tubular constriction at apex, sides gently rounded, posterior margin shallowly bisinuate, at middle $1.8 \mathrm{x}$ as broad as at apex, $0.95 \mathrm{x}$ as broad as at base, dorsum gently convex 


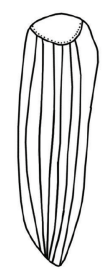

(a)

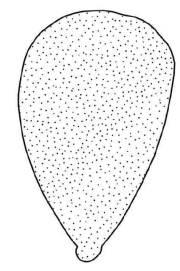

(e)

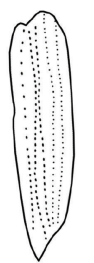

(i)

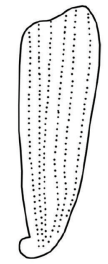

(b)

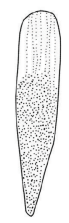

(f)

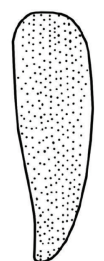

(j)

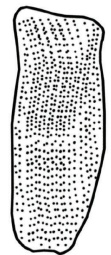

(c)

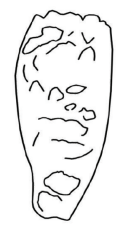

(g)

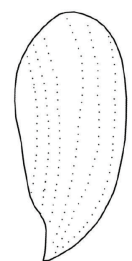

(k)

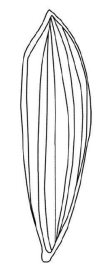

(d)

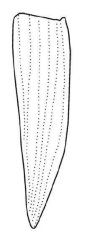

(h)

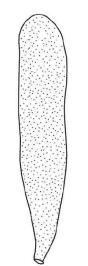

(1)

$0.0125 \mathrm{~mm}$

Figure 9: Elytral vestiture: (a), A. cyperi; (b)-(c), A. immaculata; (d)-(e), A. meghalayensis sp. nov.; (f)-(g), A. oryzae; (h)-(j), A. richardi sp. nov.; (k)-(l), A. spinulatus sp. nov.

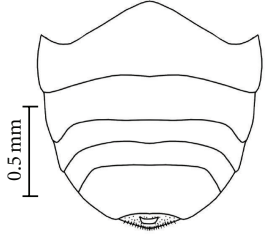

(a)

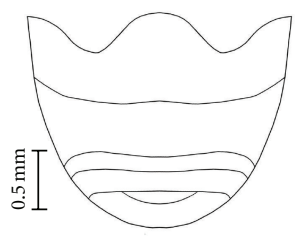

(b)

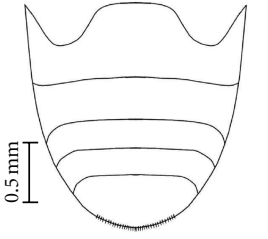

(c)

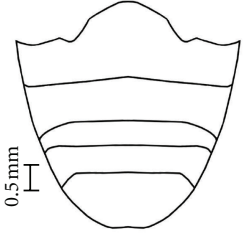

(d)

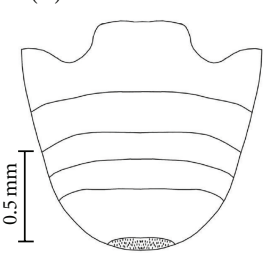

(e)

Figure 10: Venter: (a), A. cyperi; (b), A. meghalayensis sp. nov.; (c), A.oryzae; (d), A. richardi sp. nov.; (e), A. spinulatus sp. nov.

longitudinally, set with close granular punctures, confluent in curves, with smooth median line, patches of yellowish white vestiture just above posterior margin of prothorax (Figure 5(c)). Scutellum strongly transverse, square shaped, not punctate, without median impression, as broad as long. Elytra ovate, without deep subapical impressions, without posterior calli, apices rounded, at middle $1.14 \mathrm{x}$ as broad as at apex, $1.33 \mathrm{x}$ as broad as at base; striae shallow, with distant separate punctures which do not encroach on intervals, striae 10 complete; intervals $4 \mathrm{x}$ as broad as a striae, with a row of catenulate transverse punctures, each containing a minute black recumbent scale, lateral margin smooth at apex, interval five with a patch of yellowish vestiture on basal end, middle of elytra with larger patch of vestiture on interval 4 and 5 (Figure $8(\mathrm{c})$ ); elytral vestiture either brownish yellow, with basal end blunt, apex pointed with lines on surfaces reaching apex (Figure 9(d), or greyish white, pear shaped, broad at apex with granular striations on surface (Figure 9(e)). Legs coarsely punctate, each puncture containing a grey vestiture; tibiae sulcate; profemur $1.31 \mathrm{x}$ 


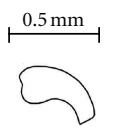

(a)

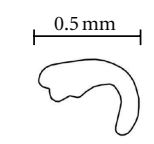

(b)

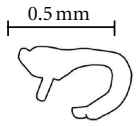

(c)

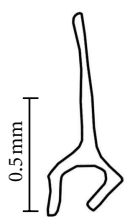

(d)

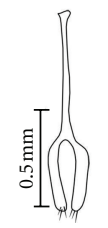

(e)

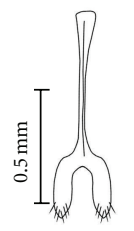

(f)

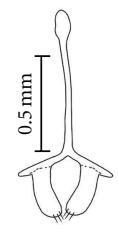

(g)

Figure 11: Female genitalia, spermatheca, and spiculum ventrale: (a), (d), A. cyperi; (b), (e), A. oryzae; (c), (f), A. richardi sp. nov.; (g), A. spinulatus sp. nov.

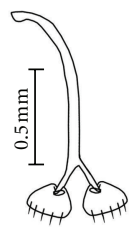

(a)

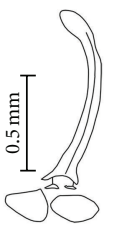

(b)

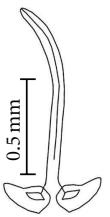

(c)

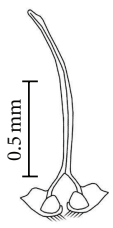

(d)

FIgUre 12: Male genitalia, spiculum gastrale: (a), A. cyperi; (b), A. meghalayensis sp. nov.; (c), A. richardi sp. nov.; (d), A. spinulatus sp. nov.

as long as mesofemur, $0.92 \mathrm{x}$ as long as metafemur. Protibia $0.97 \mathrm{x}$ as long as mesotibia, and as long as metatibia. First tarsal segment as long as broad, $1.25 \mathrm{x}$ as long as second, $0.83 \mathrm{x}$ as long as third, third $1.66 \mathrm{x}$ as broad as long, fourth $2.75 \mathrm{x}$ as long as broad. Prosternum with deep transverse sulcus behind apex, base of prosternum with raised fovea. Procoxa $1.98 \mathrm{x}$ as broad as intercoxal process; mesosternum plate like, depressed at base and apex, intercoxal process 1.03x as broad as mesocoxa; metasternum shallowly depressed at middle with impressed longitudinal line, intercoxal process $2.01 \mathrm{x}$ as broad as metacoxa. Venter black, clothed with broad greyish yellow vestiture, strongly punctate, each with broad white scale, anterior margin of first ventrite ogival, posterior margin subtruncate, $2.7 \mathrm{x}$ as broad as long, $1.66 \mathrm{x}$ as long as second, second $4.22 \mathrm{x}$ as broad as long, $1.12 \mathrm{x}$ as long as three and four combined, third and fourth subequal in length, ventrite five $3.12 \mathrm{x}$ as broad as long, posterior margin rounded; pygidium distinctly punctate, broadly exposed with long hairs from each puncture, $1.53 \mathrm{x}$ as broad as long (Figure 10(b)).

Male Genitalia. Apophyses 1.06x as long as median lobe, $0.7 \mathrm{x}$ as long as spiculum gastrale, $1.84 \mathrm{x}$ as long as tegmen; median lobe moderately sclerotised, parallel sided from base to behind middle, apex truncate, at middle $1.25 \mathrm{x}$ as broad as at apex, and 1.42x as broad at base (Figures 13(d)-13(f) and $15(\mathrm{e})-15(\mathrm{~g}))$. Tegmen $1.9 \mathrm{x}$ as long as manubrium, $1.58 \mathrm{x}$ as long as parameroid lobe; manubrium flat (Figures $13(\mathrm{~g})$ and 15(i)). Spiculum gastrale uniformly thick, curved at apex, with a median line, $12.5 \mathrm{x}$ as long as broad (Figures 12 (b) and $15(h))$.
Measurements of Holotype. Total length: $4.20 \mathrm{~mm}$; standard length: $3.80 \mathrm{~mm}$; breadth: $1.78 \mathrm{~mm}$.

Material Examined. Holotype $\sigma^{7}$, India: Meghalaya: Tura, date and coll. unknown, from wild plant (latitude: $25^{\circ} 30^{\prime}$ $\mathrm{N}$; Longitude: $\left.90^{\circ} 16^{\prime} \mathrm{E}\right)$. Paratypes $\left(2 \sigma^{7} \sigma^{7}\right): 1 \sigma^{7}$, India: Meghalaya: Ambashi, from wild plant, 26.v.1988, Coll. D. Kumar; $10^{7}$, Assam: Nagora, from wild plants, 23.v.1988, Coll. Baljinder.

Distribution. India: Meghaya; Assam.

Etymology. The specific epithet refers to the type locality.

5.4. Athesapeuta oryzae Marshall, 1916 [4]. (Figures 2(d), 3(d), 4(d), 5(d), 6(c), 8(d), 9(f), 9(g), 10(c), 11(b), 11(e), 14(c), 14(d)).

Description. Colour shiny black. Head convex, finely shagreened with regular close puncture, $1.75 \mathrm{x}$ as broad as long; eyes $1.47 \mathrm{x}$ as wide as long. Rostrum $0.92 \mathrm{x}$ as long as head and pronotum combined, strongly curved, without any subbasal dilation, with coarse punctures especially at sides, and with an impunctate median carinae, at middle $0.83 \mathrm{x}$ as broad as at apex, $0.67 \mathrm{x}$ as broad as at base (Figures 2 (d) and 3(d)). Antennae black, with whorls of stout yellowish vestiture, inserted at 1.1x of length from base of rostrum; scape slender, $10 \mathrm{x}$ as long as broad; funicle with first segment $1.42 \mathrm{x}$ as long as second and third combined, third as long as broad, segments three to seven transverse and subequal in length and breadth; funicle $1.85 \mathrm{x}$ as long as 


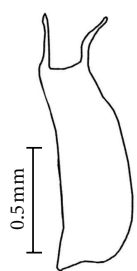

(a)

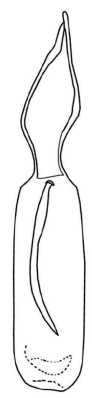

(h)

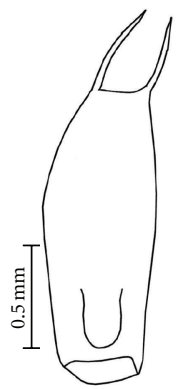

(1)

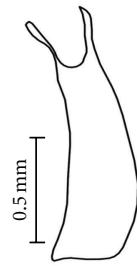

(b)

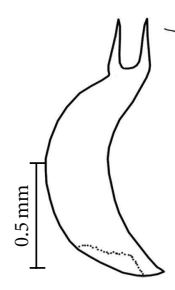

(c)

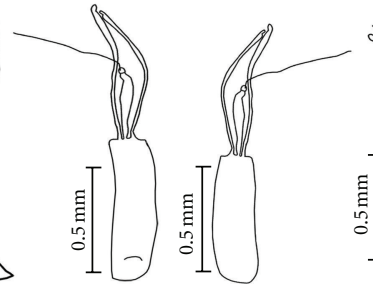

(d) (e)

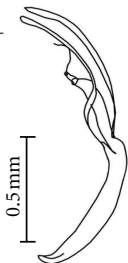

(f)

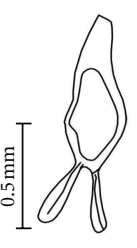

(g)

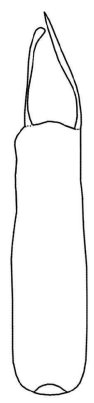

(i)

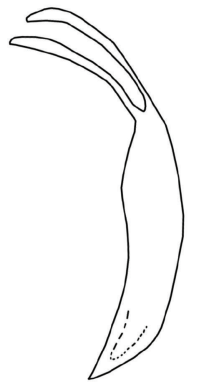

(j)

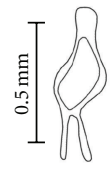

(k)

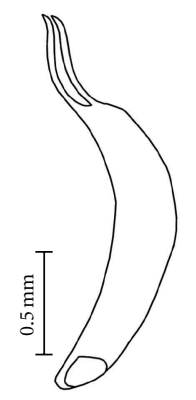

(n)

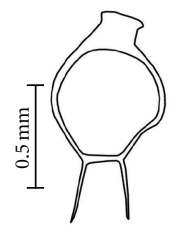

(o)

Figure 13: Median lobe (dorsal, ventral, and lateral view) and tegmen: (a)-(c), A. cyperi; (d)-(g), A. meghalayensis sp. nov.; (h)-(k), A. richardi sp. nov.; (1)-(o), A. spinulatus sp. nov.

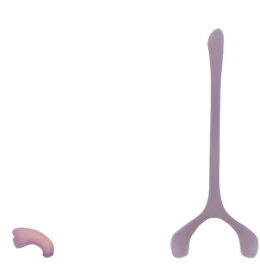

(a) (b)

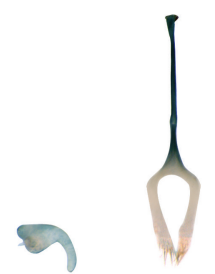

(c)

(d)

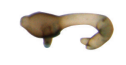

(e)

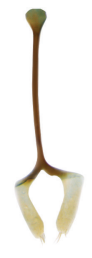

(f)

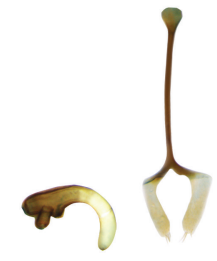

(g)

(h)

Figure 14: Female genitalia, spermatheca, and spiculum ventrale: (a), (b), A. cyperi; (c), (d), A. oryzae; (e), (f), A. richardi sp. nov.; (g), (h), A. spinulatus sp. nov.

club; club with whorls of vestiture (Figure 4(d)). Prothorax $1.19 \mathrm{x}$ as broad as long, anterior margin truncate, posterior margin bisinuate, at middle $1.9 \mathrm{x}$ as broad as at apex, $0.97 \mathrm{x}$ as broad as at base, with broad lateral stripe, which is continuous with scaling of lower surface, interrupted about middle by a small bare kidney-shaped spot, with shallow constriction at apex, gently rounded at sides, set with close coarse punctures, with an abbreviated impunctate median line (Figure 5(d)). Scutellum strongly transverse, coarsely punctate, with narrow vestiture, $1.2 \mathrm{x}$ as broad as long. Elytra oblong, separately rounded at apex, at middle $1.21 \mathrm{x}$ as broad as at apex, $1.21 \mathrm{x}$ as broad as at base, with a large basal patch consisting of lines of vestiture on intervals three to eight, those on fifth and sixth longest, those on four, three, 


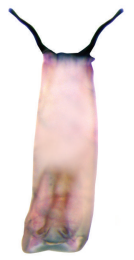

(a)

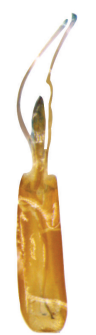

(e)

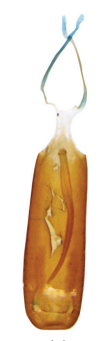

(j)

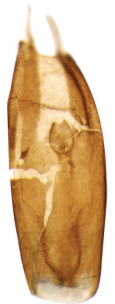

(n)

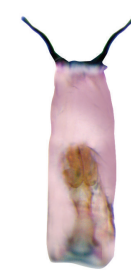

(b)

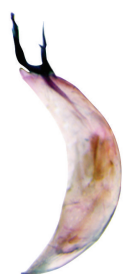

(c)

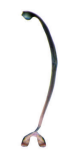

(d)

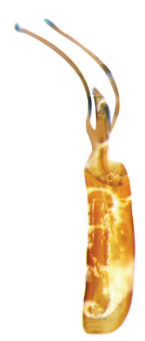

(f)

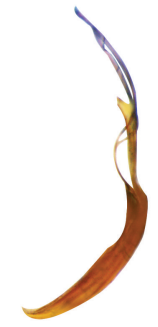

(g)

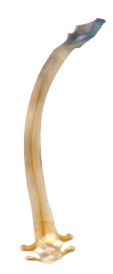

(h)

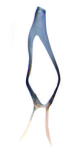

(i)

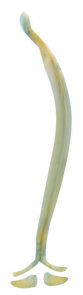

(l)

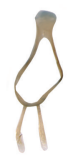

(m) (k)

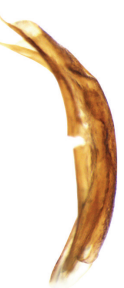

(o)

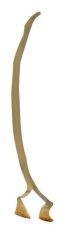

(p)

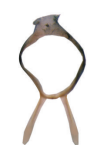

(q)

Figure 15: Male genitalia, median lobe (ventral, dorsal, and lateral view), spiculum gastrale, and tegmen: (a)-(d), A. cyperi; (e)-(i), A. meghalayensis sp. nov.; (j)-(m), A. richardi sp. nov.; (n)-(q), A. spinulatus sp. nov.

seven, and eight diminishing in the order given, whole patch covering about one-third of elytra and leaving shoulder bare, close behind it another large irregular patch, lines of which intervals 2, 3, 4, 8, and 9 are short, with 5, 6, and 7 being longer, that on 5 longest, with a "v"-shaped apical patch extending from interval 3 to 8 ; striae deep, striae 10 complete; intervals almost plane, $4 \mathrm{x}$ as broad as a striae (Figure $8(\mathrm{~d})$ ); elytral vestiture elongate, and with sparse punctures on it (Figure 9(f)), or with irregular patches on it (Figure 9(g)). Legs with densely clothed yellowish white vestiture; all tibiae at apical end on sides with fringes of hairs (Figure 6(c)); profemur $1.13 \mathrm{x}$ as long as mesofemur, $1.09 \mathrm{x}$ as long as metafemur. Protibia $1.25 \mathrm{x}$ and $1.12 \mathrm{x}$ as long as meso and metatibia respectively. First tarsal segment $0.85 \mathrm{x}$ as long as broad, $0.75 \mathrm{x}$ as long as second, $0.46 \mathrm{x}$ as long as and $0.53 \mathrm{x}$ as broad as third, third as long as broad, fourth $3.75 \mathrm{x}$ as long as broad. Prosternum with deep transverse sulcus behind apex, whole lower surface densely scaled, base of prosternum with fovea. Procoxa $2.42 \mathrm{x}$ as broad as intercoxal process; mesosternum plate like, raised at apex, intercoxal process $0.9 \mathrm{x}$ as broad as mesocoxa; metasternum flat without median line, vestiture closer on meta-episternum, intercoxal process $2 \mathrm{x}$ as broad as metacoxa. Venter black, strongly punctate, each with broad scale, anterior margin of first ventrite ogival, posterior margin shallowly straight, $3.73 \mathrm{x}$ as broad as long, $1.03 \mathrm{x}$ as long as second, second $4.5 \mathrm{x}$ as broad as long, $0.92 \mathrm{x}$ as long as three and four combined, third and fourth subequal in length, ventrite five $5 \mathrm{x}$ as broad as long, posterior margin rounded; pygidium indistinctly punctate, exposed on dorsal side, $1.33 \mathrm{x}$ as broad as long (Figure 10(c)). 


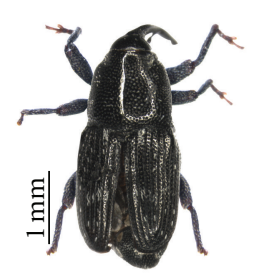

(a)
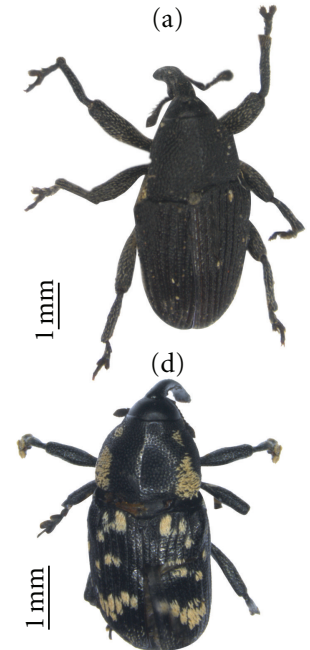

(g)

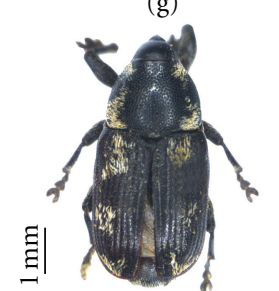

(j)

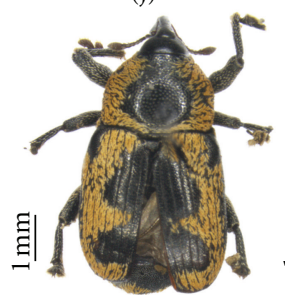

(m)

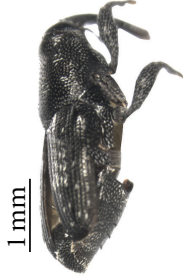

(b)

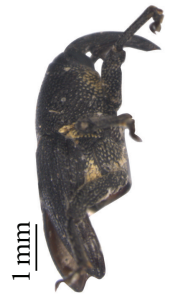

(e)

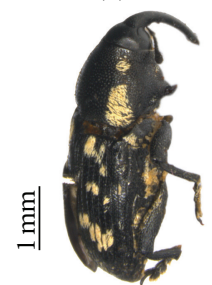

(h)

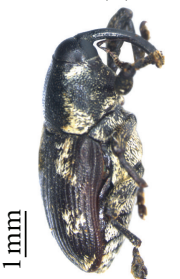

(k)

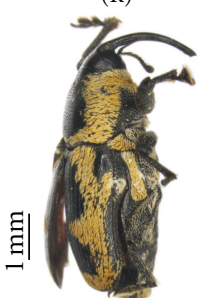

(n)

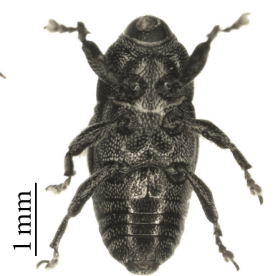

(c)

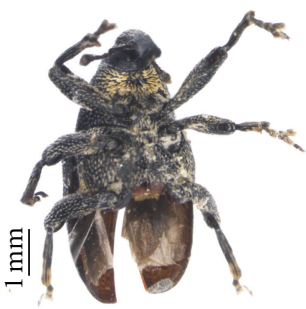

(f)

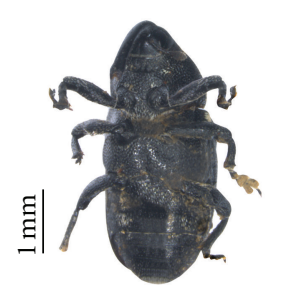

(i)

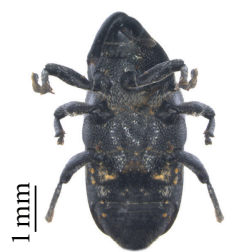

(1)

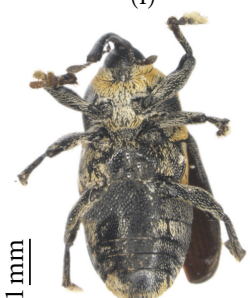

(o)

Figure 16: Habitus: dorsal, lateral, and ventral view: (a)-(c), A. cyperi; (d)-(f), A. immaculata; (g)-(i), A. meghalayensis sp. nov.; (j)-(l), A. richardi sp. nov.; (m)-(o), A. spinulatus sp. nov.

Female Genitalia. Spermatheca not sclerotised, distal arm as long as and as broad as proximal arm, angle between proximal and distal arms not acute, nodulus small, ramus flat, cornu slightly bent, not pointed (Figures $11(\mathrm{~b})$ and $14(\mathrm{c}))$. Spiculum ventrale with shaft elongate, $1.84 \mathrm{x}$ as long as basal plate, basal plate $4.75 \mathrm{x}$ as long as broad, apical end rounded with hairs (Figures 11(e) and 14(d)).

Measurements. Total length: $5.38 \mathrm{~mm}$; standard length: $4.90 \mathrm{~mm}$; breadth: $2.28 \mathrm{~mm}$.

Material Examined. 1 o, Coimbatore, Coll. and host unknown (written as Paratype and determined by G. A. K. Marshall).
Distribution. India: Tamil Nadu; Andhra Pradesh.

5.5. Athesapeuta richardi sp. nov. (Figures 2(e), 3(e), 4(e), 5(e), 7(a), 7(b), 8(e), 9(h)-9(j), 10(d), 11(c), 11(f), 12(c), 13(h)-13(k), 14(e), 14(f), 15(j)-15(m), and 16(j)-16(l)).

Diagnosis. This species is closely allied to Athesapeuta oryzae but differs in vestiture which is greyish white as compared to yellowish in A. oryzae; rostrum which is without impunctate median line, whereas in A. oryzae it is with impunctate median line; tibia with a sharp tooth just above mucro on lateral side and this is absent in A. oryzae, and female with cornu of spermatheca having finger-like projection in this species. 
Description. Colour shiny blackish yellow. Head with close punctures, $1.76 \mathrm{x}$ as broad as long; eyes ventrally placed, $1.53 \mathrm{x}$ as wide as long. Rostrum $0.80 \mathrm{x}$ as long as head and pronotum combined, strongly curved, gradually widening, punctate, without median line, almost parallel sided, without any subbasal dilation, at middle $0.92 \mathrm{x}$ as broad as at apex, $0.63 \mathrm{x}$ as broad as at base (Figures 2(e) and 3(e)). Antennae inserted at $1.68 \mathrm{x}$ of length from base of rostrum; scape robust, $8.45 \mathrm{x}$ as long as broad, almost impunctate; funicle with first segment $0.76 \mathrm{x}$ as long as second and third combined, third subequal in length and breadth, segments three to seven transverse, subequal in length and breadth; funicle $2.56 \mathrm{x}$ as long as club; club ovate (Figure $4(\mathrm{e})$ ). Prothorax $1.17 \mathrm{x}$ as broad as long, with granular punctures, with a broad lateral stripe of white vestiture, which is continuous with scaling of lower surface, interrupted about middle by a small bare kidney-shaped spot, apex with tubular constriction, sides gently rounded, anterior margin truncate, posterior margin shallowly bisinuate; at middle $1.80 \mathrm{x}$ as broad as at apex, $0.96 \mathrm{x}$ as broad as at base; dorsum gently convex longitudinally, set with close granular punctures, not confluent in curves, without any smooth median line (Figure 5(e)). Scutellum strongly transverse, square shaped, not punctate, without median impression, $1.5 \mathrm{x}$ as broad as long. Elytra ovate, without deep subapical impressions and posterior calli absent, apices rounded; at middle $1.62 \mathrm{x}$ as broad as at apex, $1.63 \mathrm{x}$ as broad as at base; striae shallow, with distant separate punctures which do not encroach on intervals, striae 10 complete; intervals $5.45 \mathrm{x}$ as broad as striae, with a row of irregular transverse punctures, each containing a minute vestiture, lateral margin smooth at apex (Figure 8(e)); elytral vestiture either white, with granular serrations reaching apex (Figure 9(h)), or brown with serrated granular ridges on it (Figure 9(i)), or surface without regular serrations but with scattered punctures (Figure $9(j)$ ). Legs coarsely punctate, each puncture containing a grey vestiture; tibiae sulcate, posterior end of all tibiae just before mucro carries sharp tooth (Figure $7(\mathrm{a})$ ); metatibia with fringes of grey hairs on lateroventral side (Figure 7(b)); profemur 1.06x as long as mesofemur, $0.93 \mathrm{x}$ as long as metafemur. Protibia $1.19 \mathrm{x}$ and $1.07 \mathrm{x}$ as long as meso- and metatibia, respectively. First tarsal segment $1.35 \mathrm{x}$ as long as broad, $0.98 \mathrm{x}$ as long as second, $0.76 \mathrm{x}$ as long as third, third $1.24 \mathrm{x}$ as broad as long, fourth $4.80 \mathrm{x}$ as long as broad. Prosternum with deep transverse sulcus behind apex, base with raised fovea. Procoxa 1.98x as broad as intercoxal process; mesosternum plate like, depressed at base and apex, intercoxal process $1.03 \mathrm{x}$ as broad as mesocoxa; metasternum shallowly depressed in middle with longitudinal line, intercoxal process $2.01 \mathrm{x}$ as broad as metacoxa. Venter black, strongly punctate, each with broad white vestiture, anterior margin of first ventrite ogival, posterior margin subtruncate, $3.09 \mathrm{x}$ as broad as long, $1.64 \mathrm{x}$ as long as second, second $4.70 \mathrm{x}$ as broad as long, $0.89 \mathrm{x}$ as long as three and four combined, third and fourth subequal in length, ventrite five $3.48 \mathrm{x}$ as broad as long, posterior margin rounded; pygidium with indistinct punctures, $1.53 \mathrm{x}$ as broad as long (Figure 10(d)).
Female Genitalia. Spermatheca more sclerotised at proximal arm, distal arm $0.93 \mathrm{x}$ as long as proximal arm, angle between proximal and distal arms acute, nodulus small, tapering towards apex, ramus tubular and long, cornu strongly pointed and with a finger-like projection (Figures $11(\mathrm{c})$ and $14(\mathrm{e}))$. Spiculum ventrale with shaft elongate, $2 \mathrm{x}$ as long as basal plate, basal plate $3 \mathrm{x}$ as long as broad, apical end pointed with hairs (Figures 11(f) and 14(f)).

Male Genitalia. Apophyses $1.06 \mathrm{x}$ as long as median lobe, $0.7 \mathrm{x}$ as long as spiculum gastrale, $1.84 \mathrm{x}$ as long as tegmen; median lobe moderately sclerotised, parallel sided from base to behind middle, apex truncate, at middle $1.25 \mathrm{x}$ as broad as at apex, and $1.42 \mathrm{x}$ as broad as at base (Figures 13(h)$13(\mathrm{k}), 15(\mathrm{j})$ and $15(\mathrm{k}))$. Tegmen $1.9 \mathrm{x}$ as long as manubrium, $1.58 \mathrm{x}$ as long as parameroid lobe, manubrium flat (Figures $13(\mathrm{k})$ and $15(\mathrm{~m}))$. Spiculum gastrale uniformly thick, curved at apex, with a median line, $12.5 \mathrm{x}$ as long as broad (Figures 12(c) and 15(1)).

Measurements of Holotype. Total length: $5.42 \mathrm{~mm}$; standard length: $5.20 \mathrm{~mm}$; breadth: $2.30 \mathrm{~mm}$.

Material Examined. Holotype $\sigma^{x}$ : India: Meghalaya: Tura, Date and Coll. unknown, from wild plant (latitude: $25^{\circ} 30^{\prime}$ N; longitude: $\left.{ }^{\circ} 16^{\prime} \mathrm{E}\right)$. Paratypes: 16 specimens $\left(2 \sigma^{7} \sigma^{7}, 1\right.$ o $)$ : India: $2 \sigma^{\top}$, Meghalaya: Tura, date and coll. unknown, from wild plant; 1 o, 12 specimens, Andhra Pradesh: Patancheru, 18.ix.1985, Coll. M.Haq, from agricultural plants.

Distribution. India: Meghalaya; Andhra Pradesh.

Etymology. The name is derived from and in recognition of Dr. Richard Thompson for his contribution towards baridine weevils.

5.6. Athesapeuta spinulatus sp. nov. (Figures 2(f), 3(f), 4(f), 5(f), 8(f), 9(k), 9(1), 10(e), 11(g), 12(d), 13(1)-13(o), 14(g), 14(h), 15(n)-15(q), and 16(m)-16(o))

Diagnosis. This species is closely related to A. immaculata but differs in its larger size, with dense vestitures all over body; antennae with all funicular segments with four rows of spines; elytra ovate (oblong ovate in immaculata).

Description. Colour black. Head with shallow sparse punctures, $1.8 \mathrm{x}$ as broad as long; eyes ventrally placed, $1.48 \mathrm{x}$ as wide as long. Rostrum $0.91 \mathrm{x}$ as long as head and pronotum combined, curved, gradually widening, broadest at apex, closely punctate at basal side on lateral aspect, each puncture with yellow vestiture, dorsal surface smooth, at middle $0.75 \mathrm{x}$ as broad as apex, 0.66x as broad as at base, without any subbasal dilation (Figures 2(f) and 3(f)). Antennae inserted at 1.36x of length from base of rostrum; scape slender, long, $8.75 \mathrm{x}$ as long as broad, almost impunctate; funicle with first segment $1.1 \mathrm{x}$ as long as second and third combined, third $1.3 \mathrm{x}$ as long as broad, segments three to seven transverse 
and subequal in length and breadth; funicle $2.53 \mathrm{x}$ as long as club, all segments carry sharp four rows of spines on its surface, seventh funicular segment broadest, which is not in continuous with club; club ovate (Figure 4(f)). Prothorax $1.28 \mathrm{x}$ as broad as long, with granular punctures at centre, a stripe of yellow vestiture on lateral aspect on both sides, which is continuous with scaling of lower surface, without tubular constriction at apex, broadly rounded at sides, anterior margin truncate, posterior margin shallowly bisinuate, at middle $2.22 \mathrm{x}$ as broad as at apex, $1.03 \mathrm{x}$ as broad as at base, dorsum gently convex longitudinally without any median line (Figure 5(f)). Scutellum strongly transverse, not punctate, without median impression, $1.5 \mathrm{x}$ as broad as long. Elytra ovate, without subapical impressions and without posterior calli, apices rounded, at middle $1.2 \mathrm{x}$ as broad as at apex, $1.5 \mathrm{x}$ as broad as at base; striae shallow, with distant separate punctures which do not encroach on intervals, striae 10 complete; intervals $6 \mathrm{x}$ as broad as a striae, with a row of catenulate transverse punctures, each containing a minute black recumbent vestiture, lateral margin smooth at apex, a patch of yellowish vestiture starts from interval 2 which continues up to 10 (Figure 8(f)); elytral vestiture yellow, either with broad parallel striae running from base to apex (Figure $9(\mathrm{k})$ ), or with granular striations on surface (Figure 9(1)). Legs coarsely punctate, each puncture containing a grey vestiture; tibiae sulcate; profemur as long as mesofemur, $0.8 \mathrm{x}$ as long as metafemur. Protibia $1.25 \mathrm{x}$ and $1.1 \mathrm{x}$ as long as meso- and metatibia, respectively. First tarsal segment $1.66 \mathrm{x}$ as long as broad, $1.5 \mathrm{x}$ as long as second, and as long as third, third $1.66 \mathrm{x}$ as broad as long, fourth $6.66 \mathrm{x}$ as long as broad. Prosternum with deep transverse sulcus behind apex, base of prosternum with raised fovea. Procoxa $1.53 \mathrm{x}$ as broad as intercoxal process; mesosternum plate like, depressed at base and apex, intercoxal process $1.1 \mathrm{x}$ as broad as mesocoxa; metasternum shallowly depressed in middle with impressed longitudinal line, intercoxal process $2.05 \mathrm{x}$ as broad as metacoxa. Venter black, clothed with broad greyish yellow vestiture, strongly punctate and each with broad vestiture, anterior margin of first ventrite sinusoidal, posterior margin subtruncate, $2.91 \mathrm{x}$ as broad as long, $1.72 \mathrm{x}$ as long as second, second $4.75 \mathrm{x}$ as broad as long, $0.75 \mathrm{x}$ as long as three and four combined, third and fourth subequal in length, ventrite five $2.48 \mathrm{x}$ as broad as long, its posterior margin rounded; pygidium distinctly punctate, broadly exposed with long hairs from each puncture, 1.02x as broad as long (Figure 10(e)).

Female Genitalia. Spermatheca more sclerotised at proximal arm, distal arm as long as proximal arm, angle between proximal and distal arms acute, nodulus small, tapering towards apex, ramus small, cornu bent and blunt at apex (Figure $14(\mathrm{~g})$ ). Spiculum ventrale with shaft elongate, $2.5 \mathrm{x}$ as long as basal plate, basal plate $2.85 \mathrm{x}$ as long as broad, apical end truncate with hairs, basal end with a lateral projection (Figures 11(g) and 14(h)).

Male Genitalia. Apophyses $0.44 \mathrm{x}$ as long as median lobe, $0.4 \mathrm{x}$ as long as spiculum gastrale, as long as tegmen; median lobe moderately sclerotised, parallel sided from base to behind middle, apex truncate, at middle $1.9 \mathrm{x}$ as broad as at apex, 1.35x as broad at base (Figures 13(1)-13(n), 15(n), and $15(\mathrm{o}))$. Tegmen $2.85 \mathrm{x}$ as long as manubrium, $2 \mathrm{x}$ as long as parameroid lobe, manubrium short and flat (Figures 13(o) and 15(q)). Spiculum gastrale uniformly thick, not curved at apex, with a median line, 16.66x as long as broad (Figures 12(d) and 15(p)).

Measurements of Holotype. Total length: $5.69 \mathrm{~mm}$; standard length: $5.30 \mathrm{~mm}$; breadth: $2.33 \mathrm{~mm}$;

Material Examined. Holotype $0^{7}$ : India: Haryana: Jind, 9.vii.1986, coll. unknown, from grass (latitude: $29^{\circ} 48^{\prime} \mathrm{N}$; longitude: $78^{\circ} 26^{\prime}$ E). Paratypes ( $20^{\top} \sigma^{\prime \prime}, 2$ 우): India: 1 우 Haryana: Jind, 9.vii.1986, coll. unknown, from grass; $20^{7} 0^{7}$, Andhra Pradesh: Patancheru, 18.ix.1985, coll. unknown, from wild aquatic plant; 1 o (no data).

Distribution. India: Haryana; Andhra Pradesh.

Etymology. The specific name is given after the funicular segments which have sharp spines in four rows.

\section{Key to the Indian Species of Athesapeuta}

(1) (a) Rostrum without carinae-2.

(b) Rostrum with carinae-3.

(2) (a) Rostrum with four rows of small punctures; prothorax with a broad impunctate median stripe; profemur with fringes of long vestiture on ventral surface (Figure 6(a)); protibia with sharp tooth on inner edge at about middle in males (Figure 6(b)), females without it—cyperi.

(b) Rostrum without four rows of small punctures; prothorax without a broad stripe; profemur without fringes of long vestiture on ventral surface; protibia without tooth in males or females-4.

(3) (a) Tibiae at apical end on sides with fringes of hairs (Figure 6(c)); elytra oblong, with large basal patch consisting of lines of vestiture on intervals three to eight, those on fifth and sixth longest, those on four, three, seven, and eight diminishing in the order given, whole patch covering about one third of the elytra and leaving the shoulder bare, close behind it another large irregular patch, lines of which intervals $2,3,4,8$, and 9 are short, and 5, 6 and 7 being longer, that on 5 longest, with a " $\mathrm{v}$ "-shaped apical patch extending from interval 3 to 8 -oryzae.

(b) Tibiae without fringes of hairs; elytra not oblong but ovate or oblongovate- 5 .

(4) (a) Funicular segments without spines; posterior end of all tibiae carries sharp tooth (Figure 7(a)), metatibia with fringes of grey hairs on lateroventrally (Figure 7(b)); apex of prothorax with tubular constriction, granular punctures all over, sides gently 
rounded; apophyses $1.06 \mathrm{x}$ as long as median lobe; spiculum gastrale $12.5 \mathrm{x}$ as long as broad-richardi, sp. nov.

(b) Funicular segments with spines (Figure 4(f)); posterior end of all tibia does not carry sharp tooth; apex of prothorax without tubular constriction, granular punctures only at centre, sides broadly rounded; apophyses $0.44 \mathrm{x}$ as long as median lobe; spiculum gastrale $16.66 \mathrm{x}$ as long as broadspinulatus, sp. nov.

(5) (a) Prothorax with broad lateral stripe, with smooth median line; elytra ovate (Figure $8(\mathrm{c})$ ); elytal vestiture either brownish yellow, with basal end blunt, apex pointed with lines on surfaces reaching apex (Figure 9(d)), or greyish white, pear shaped, broad at the apex with granular striations on the surface (Figure 9(e))—meghalayensis, sp. nov.

(b) Prothorax without broad lateral stripe, without median line; elytra oblongovate (Figure 8(b)); elytral vestiture either yellowish white, base tapering, apex concave, surface with granular serrations (Figure 9(b)), or brownish yellow, both ends broader, surface with dense granular serrations (Figure 9(c))_immaculata.

\section{Acknowledgment}

Senior author gratefully acknowledges the Kerala Agricultural University for providing deputation for study purpose.

\section{References}

[1] M. A. Alonso-Zarazaga and C. H. C. Lyal, "A world catalogue of families and genera of Curculionoidea (Insecta: Coleoptera) (excepting Scolytidae and Platypodidae)," Entomopraxis, S.C.P., Barcelona, Spain, pp. 1-315, 1999.

[2] J. Faust, "Viaggio di Leonardo Fea in Birmania e regioni vicine. LX. Curculionidae," Annali del Museo Civico di Storia Naturale di Genova, vol. 34, pp. 153-370, 1894.

[3] J. Faust, "Beschreibung neuer Coleopteren von Vorder-und Hinterindien aus der Sammlung des Hrn. Andrewes in London. Curculionidae," Deutsche Entomologische Zeitschrift, no. 2, pp. 273-333, 1898.

[4] G. A. K. Marshall, "Some injurious Indian Weevils (Curculionidae)-II," Bulletin of Entomological Research, vol. 6, pp. 365-373, 1916.

[5] N. R. Supare, S. Ghai, and V. V. Ramamurthy, "A revision of Tanymecus from India and adjacent countries (Coleoptera: Curculionidae)," Oriental Insects, vol. 24, pp. 1-126, 1990.

[6] V. V. Ramamurthy and S. Ghai, "A study on the genus Myllocerus (Coleoptera: Curculionidae)," Oriental Insects, vol. 22, pp. 377-500, 1988.

[7] R. T. Thompson, "Observations on the morphology and classification of weevils (Coleoptera, Curculionoidea) with a key to major groups," Journal of Natural History, vol. 26, no. 4, pp. 835-891, 1992.

[8] J. Poorani and V. V. Ramamurthy, "Weevils of the genus Lepropus Schoenherr from the oriental region (Coleoptera: Curculionidae: Entiminae)," Oriental Insects, vol. 31, pp. 1-82, 1997.
[9] C. W. O'Brien and J. Pakaluk, "Two new species of Acythopeus Pascoe (Coleoptera: Curculionidae: Baridinae) from Coccinia grandis (L.) Voight (Cucurbitaceae) in Kenya," Proceedings of the Entomological Society of Washington, vol. 100, no. 4, pp. 764-774, 1998.

[10] M. Wanat, "Alignment and homology of male terminalia in Curculionoidea and other Coleoptera," Invertebrate Systematics, vol. 21, no. 2, pp. 147-171, 2007.

[11] S. R. Davis, "Morphology of Baridinae and related groups (Coleoptera, Curculionidae)," ZooKeys, vol. 10, pp. 1-136, 2009.

[12] V. de. Motschulsky, "Essai d'un catalogue des insects de l' ile de Ceylan. Supplement," Bulletin de la Societe Imperiale des Naturistes de Moscou, vol. 39, no. 1, pp. 393-446, 1866.

[13] E. Voss, "Ein Beitrage zur kenntnis der Curculioniden im Grenzgebiet der Orientalischen zur Palaarktischen Region (Col., Curc.)," Decheniana Beihefte, vol. 5, pp. 1-139, 1958, Die von J. Klapperich und Tschung Sen in der Provinz Fukien gesammelten Russelkafer.

[14] A. Hustache, "Essai d' un tableau synoptique des Zygopides d' Afrique et de Madagascar. Livre du Centenaire," Societe Entomologique de France, pp. 1-729, 1932.

[15] G. A. K. Marshall, "New curculionidae (Col.) from Uganda," The Annals and Magazine of Natural History, vol. 8, no. 11, pp. 33-48, 1941.

[16] A. Hustache, "Curculionides nouveaux de l' Afrique Equitoriale (IIe partie)," Sbornik Entomologickeho Oddeteni Narodniho Musea v Praze, vol. 10, pp. 28-109, 1932.

[17] G. A. K. Marshall, "New injurious Curculionidae (Col.)," Bulletin of Entomological Research, vol. 18, no. 3, pp. 266-257, 1928.

[18] G. A. K. Marshall, "Curculionidae (Col.) attacking cultivated plants," Bulletin of Entomological Research, vol. 27, no. 2, pp. 253-259, 1936.

[19] H. R. Pajni and S. K. Kohli, "Current status of some Indian species of genus Baris Germ. and the description of one new genus and three new species (Coleoptera; Curculionidae; Baridinae; Barini)," Research Bulletin of the Punjab University Science, vol. 41, no. 1-4, pp. 45-59, 1990.

[20] J. C. Fabricius, Supplementum Entomologiae Systematicae, Proft et Storch, Hafniae, Copenhagen, Denmark, 1798.

[21] A. Hustache, "Curculionidae: barinae," in Coleopterorum Catalogus, W. Junk and S. Schenkling, Eds., vol. 163, pp. 1219, Pars, Gravenhage, The Netherlands, 1938.

[22] J. C. Fabricius, Systema Eleutheratorum Secundum Ordines, Genera, Species: Adiectis Synonimis, Locis, Observationibus, Descriptionibus, vol. 2, Bibliopoli Academici Novi, Kiliae, 1801.

[23] C. J. Schoenherr, "Genera et species Curculionidem, cum synonymia hujus familiae. Species novae aut hactenus minus cognitae, descriptionibus a Dom," Leonardo Gyllenhal, C.H. Boheman, et entomologis aliis illustratae, vol.3, no. 2, pp. 506858, Roret, Paris, france, 1836.

[24] J. Desbrochers des loges, "Curculionides et Brenthides du Bengalee occidental recueillis par le R.P. Cardon avec description d' especes nouelles," Annales de la Societe Entomologique de Belgique, vol. 35, pp. 350-361, 1891.

[25] G. A. K. Marshall, "Entomological results from the Swedish expedition 1934 to Burma and British India-Coleoptera, Curculionidae," Novitates Zoologicae, vol. 42, no. 3, pp. 397$473,1948$. 
[26] E. Voss, "Ceylonesische Curculioniden aus dem Naturhistorischen Museum zu Basel von F. Keiser 1953/54," Verhandlungen der Naturforschenden Gesellschaft in Basel, vol. 68, no. 1, pp. 97-118, 1957.

[27] E. Voss, Attelabidae, Apionidae, Curculionidae (Coleoptera: Rhynchophora), vol. 44 of Exploration du parc National de l'Upemba. Mission G.F.de Witte 1948-49, 1962.

[28] A. Hustache, "Curculionides Rapportes de l'Angolanpar la mission scientifique Suisse," Bulletin de la Societe Neuchateloise des Sciences Naturelles, vol. 60, pp. 165-207, 1935.

[29] E. C. Zimmerman, "Insects of Guam-I. Coleoptera: Anthribidae and Curculionidae," Bullettin of the Bernice P. Bishop Museum, vol. 172, pp. 65-146, 1942.

[30] C. H. Boheman, “Coleoptera," In: P. A. Norstedt \& Soner (Eds.) Kongliga Svenska Fregatten Eugenies Resa omkring jorden under befall af C. A. Virgin Arhen 1851-1853. Vetenskapliga la Hagester pa H. M. Konung Oscar Den Forstes befallning. K. Svenska Vetenskaps Akademien. Stockholm. 1858-68. pp. 1-617, 1859. 

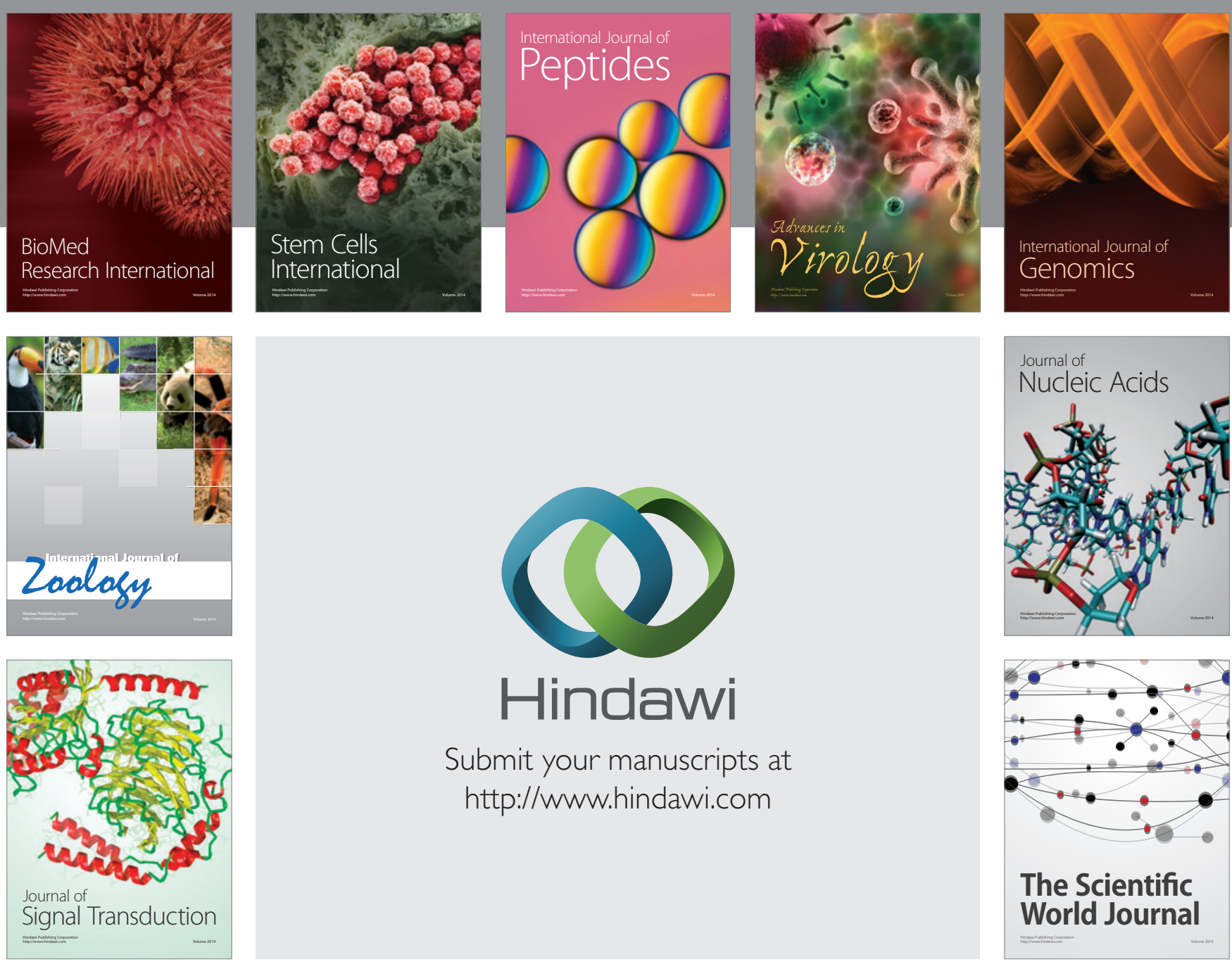

Submit your manuscripts at

http://www.hindawi.com
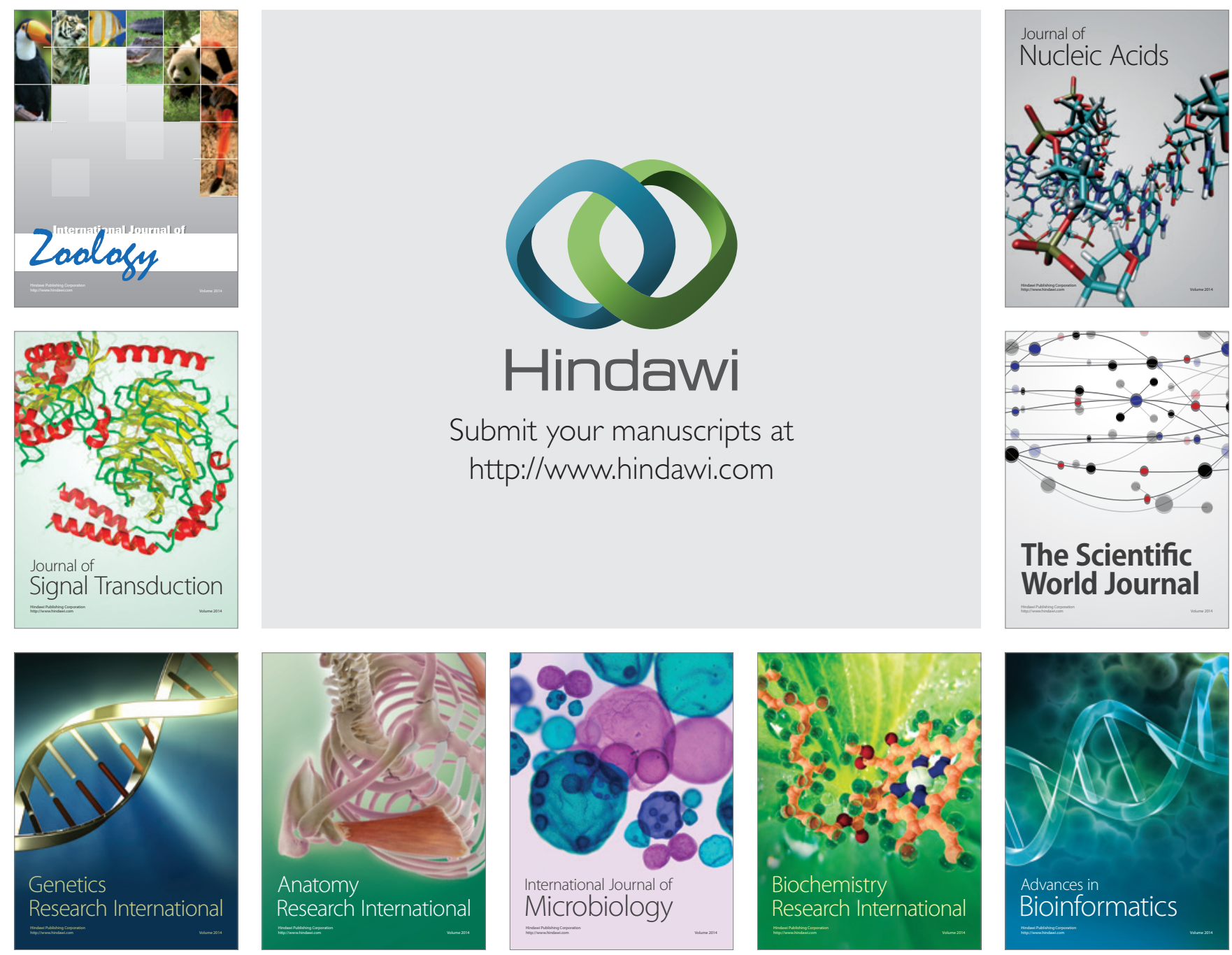

The Scientific World Journal
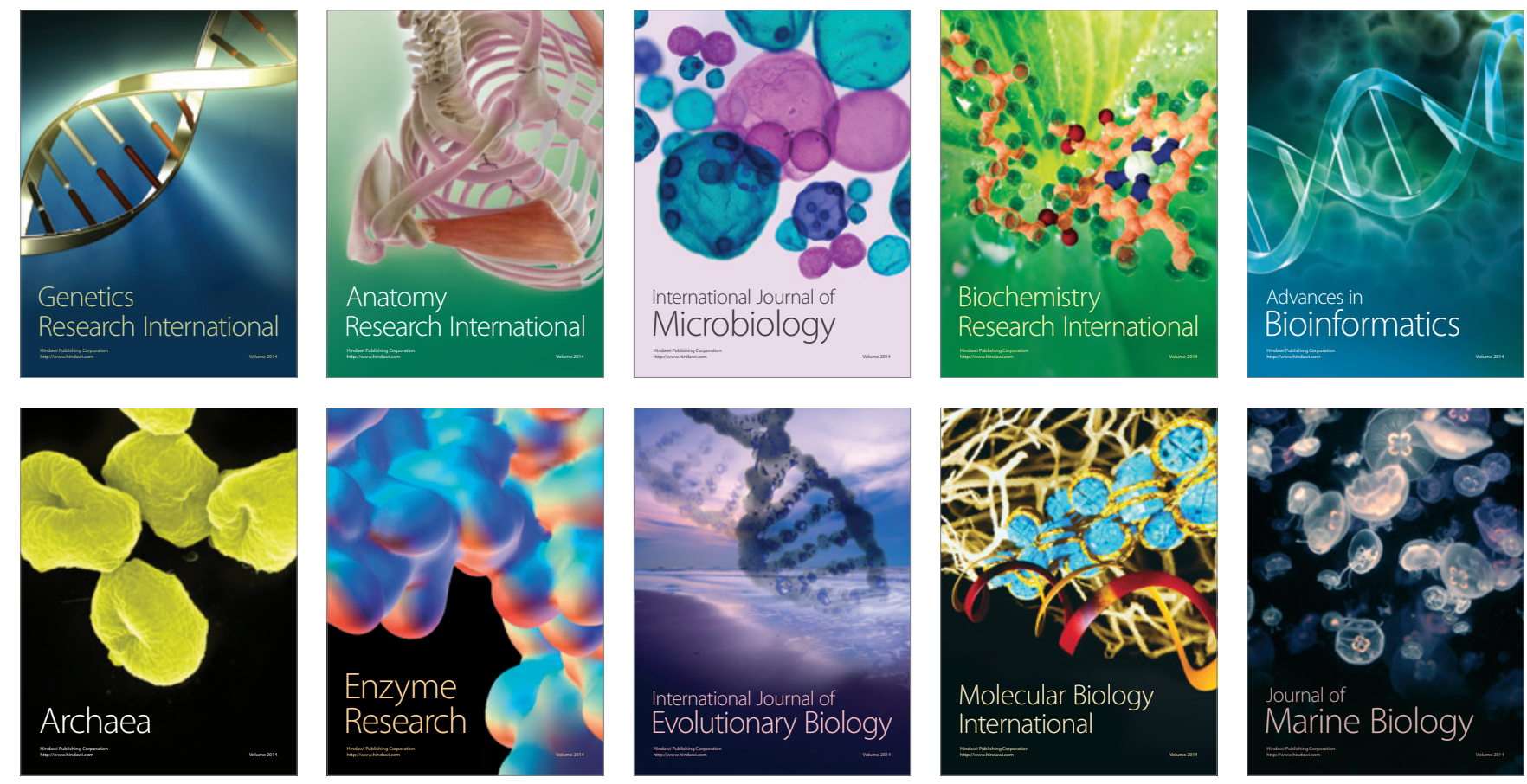